'Departamento de Medicina Interna, Programa de Geriatría, Facultad de Medicina, Pontificia Universidad Católica de Chile. 2Departamento de Salud Pública, Facultad de Medicina, Pontificia Universidad Católica de Chile.

${ }^{3}$ Departamento de Psiquiatría,

Programa de psiquiatría de enlace, Facultad de Medicina, Pontificia Universidad Católica de Chile. ${ }^{4}$ Facultad de Medicina Universidad de Antofagasta ${ }^{\mathrm{a}}$ Doctor en Ciencias PhD.

* Marcela Carrasco Gorman y Matías González Tugas participaron de igual manera en el proceso de elaboración de este trabajo y son los autores responsables de la correspondencia.

Financiamiento: Este trabajo fue financiado por un fondo concursable de la Pontificia Universidad Católica de Chile, DIPUC No 2005/15PI. No hubo participación alguna de dicha institución en ningún aspecto de la investigación.

Conflictos de interés: los autores declaran no tener conflictos de interés en relación a este artículo.

Recibido el 13 de enero de 2014 aceptado el 24 de junio de 2014

Correspondencia a: Dra. Marcela Carrasco G.

Departamento de Medicina Interna, Programa de Geriatría Pontificia Universidad Católica de Chile.

Marcoleta 350. Santiago, Chile. Fax: 5623546820 mcarras@med.puc.c

Dr. Matías González T. Departamento de Psiquiatría,

Programa de psiquiatría de enlace, Facultad de Medicina, Pontificia Universidad Católica de Chile. Lira 44, Casilla 114-D, Santiago de Chile, Chile. Fax: +562 23546820 magonza@med.puc.c

\section{Riesgo de delirium durante la hospitalización en personas mayores: desarrollo y validación de un modelo de predicción clínica}

\author{
MARCELA CARRASCO G. ${ }^{1, *}$, LUIS VILLARROEL D. ${ }^{2, a}$, \\ JORGE CALDERÓN P. ${ }^{3}$, GABRIEL MARTÍNEZ F. ${ }^{4}$, \\ MARICARMEN ANDRADE A. ${ }^{1}$, MATÍAS GONZÁLEZ T. ${ }^{3, *}$
}

\section{Development and validation of a clinical predictive model for delirium in hospitalized older people}

Background: Delirium is a prevalent problem among older patients and it is frequently underdiagnosed. Aim: To develop and validate a clinical predictive model to identify patients at high risk of delirium. Material and Methods: Two consecutive prospective cohort studies were used to develop and validate the model. The development cohort included 542 consecutive medical inpatients, 65 years or older. The validation cohort included 85 comparable patients. A predictive score was constructed with a multivariate analysis, using variables independently associated with delirium and subsequently tested in the new cohort. Patients were assessed within the first 48 hours of admission, and every 48 hours thereafter, using the Confusion Assessment Method to diagnose delirium, evaluating also the severity of underlying disease, comorbidities, functionality, and laboratory data. Results: Delirium occurred in 192 patients (35.4\%) of the development cohort and was independently associated with age and functional status assessed using the Barthel Index. With these two variables, the predictive score for delirium was developed and tested rendering an area under the receiver operating characteristic (ROC) of 0.80 (confidence intervals 0.77-0.85). Cut-off points were chosen to establish low, intermediate, and high-risk groups for delirium. According to these cut-off points, delirium frequencies in the development cohort were $8 \%, 23 \%$, and $69 \%$, and in the validation cohort $5 \%$, $34 \%$, and $66 \%$, respectively $\left(\chi^{2} p<0.05\right)$. Conclusions: This simple predictive model based on age and functional status may be a useful tool for identifying older patients risking delirium.

(Rev Med Chile 2014; 142: 826-832)

Key words: Aged; Delirium; Inpatients.
1 1 delirium, definido como un trastorno agudo y de curso fluctuante que afecta la capacidad de atención y funcionamiento cognoscitivo ${ }^{1}$, ha sido reconocido como un problema de alta relevancia, especialmente en ancianos hospitalizados. Diferentes estudios revelan que es una de las complicaciones intrahospitalarias más frecuentes, alcanzando una prevalencia de hasta $42 \%$ en pacientes hospitalizados por patologías médicas ${ }^{2-4}$.

Uno de los factores que marcan la importancia del delirium es que afecta radicalmente el pro- 
nóstico del individuo. Ha demostrado asociarse a eventos negativos que incluyen mayor riesgo de complicaciones intrahospitalarias, deterioro funcional, estadía hospitalaria, y mayor mortalidad tanto intra como extrahospitalaria ${ }^{5-8}$. A pesar de esta evidencia, ha sido difícil incorporar esta condición como un problema relevante en la clínica habitual y aún existen importantes cifras de subdiagnóstico ${ }^{9}$ y escasos programas de prevención y manejo en la práctica clínica habitual ${ }^{10}$.

El desafío actual es mejorar el pronóstico y manejo de los pacientes mayores hospitalizados, ya que si bien las estrategias de manejo sintomático del delirium ayudan a controlar la gravedad de los síntomas ${ }^{11}$, aún no han demostrado mejorar el pronóstico vital. Por otro lado, existe evidencia que sustenta que la implementación de programas de prevención del delirium logran reducir su incidencia hasta en 30\% y consiguen disminuir la duración del episodio ${ }^{12-16}$.

Los programas de prevención incluyen la necesidad de implementar estrategias que permitan identificar los pacientes en mayor riesgo de delirium. Inouye et al. desarrolló un modelo predictivo de delirium incidente en pacientes mayores de 70 años, hospitalizados por patologías médicas, sin demencia ni delirium al ingreso. En la cohorte inicial se identificaron cuatro factores de riesgo: deterioro visual, enfermedad grave, deterioro cognitivo y elevación de la relación BUN/creatinina plasmática como marcador de deshidratación ${ }^{17}$. Otro modelo enfocado en los factores precipitantes incorpora cinco factores de riesgo independientes: uso de restricción física, uso de más de tres medicamentos, malnutrición, catéter urinario y eventos iatrogénicos ${ }^{18}$. Otros modelos, como el de Pompey, emplean características demográficas, depresión, cognición, funcionalidad y alcoholismo ${ }^{19}$. O'Keeffe incluye demencia, severidad de la enfermedad y niveles de urea elevados ${ }^{20}$.

Los modelos previos han aportado información relevante en relación a los factores de riesgo y precipitantes para delirium y han sido validados en diferentes cohortes, sin embargo, ellos excluyeron pacientes que ingresaban con delirium o demencia al hospital. Este hecho reduce la aplicabilidad de estos modelos, ya que ambas condiciones son subdiagnosticadas en los servicios de agudos.

El objetivo de este estudio es identificar los principales factores asociados al desarrollo de delirium en personas mayores hospitalizadas en servicios médicos, incluyendo tanto el delirium prevalente (el que está presente en el momento del ingreso) como el incidente (el que aparece durante el período de observación). Con el fin de generar un índice de riesgo de esta complicación y validarlo en una nueva cohorte.

\section{Material y Método}

Este estudio se desarrolló en dos fases, en la primera se utilizó una cohorte "de desarrollo" para identificar los factores asociados a la presencia de delirium, con lo cual se generó un índice de riesgo de esta complicación, que fue validado en la segunda fase en una cohorte llamada "validación".

Este estudio fue aprobado por el Comité de ética de la Facultad de Medicina de la Pontificia Universidad Católica de Chile y se obtuvo el consentimiento informado de los pacientes o sus representantes previo a su enrolamiento.

\section{Cohorte de desarrollo}

Para la identificación de los factores de riesgo asociados a la presencia de delirium, se usó una cohorte de pacientes consecutivos de 65 años o más, ingresados en las últimas $48 \mathrm{~h}$ a unidades de hospitalización médica durante 6 meses. Se excluyó a pacientes con afasia, en coma, barreras lingüística e inhabilidad para participar en entrevistas de evaluación cognitiva. Las evaluaciones fueron realizadas cada $48 \mathrm{~h}$, hasta el alta o por un máximo de 12 días, por un equipo médico de psicogeriatría entrenados en la detección de delirium. Más detalles de las características de esta cohorte fueron publicadas ${ }^{8}$.

Se registró información demográfica (género, edad y datos de identificación), laboratorio (electrolitos plasmáticos, nitrógeno ureico, creatinina plasmática, proteína $C$ reactiva, albúmina plasmática); de severidad de la enfermedad mediante el índice APACHE $\mathrm{II}^{21}$, carga de comorbilidad mediante el Índice de Charlson ${ }^{22}$ y de funcionalidad previa al ingreso mediante índice de Barthel ${ }^{23}$. El índice de Barthel evalúa la capacidad del individuo para desempeñar 10 actividades de la vida diaria, dando una estimación cuantitativa del nivel de funcionalidad con valores de 0 (dependencia total) a 100 (independencia total).

El delirium fue evaluado mediante el instru- 
mento de detección "Confusion Assesment Method (CAM)" adaptado para hispanoparlantes ${ }^{24}$. En este instrumento el diagnóstico de delirium se basa en la presencia de un cambio agudo y fluctuante de las funciones cognoscitivas, inatención y la presencia de pensamiento desorganizado o bien alteración del nivel de conciencia. En caso de diagnosticarse delirium, ya sea en la visita inicial o bien en el seguimiento posterior, el paciente fue incluido en la cohorte "con delirium". Si el paciente no presentó delirium en ninguna evaluación, fue ingresado a la cohorte "sin delirium".

\section{Análisis estadístico}

Se realizó análisis univariado (pruebas t y $\chi^{2}$ ) con el fin de determinar la asociación entre las características clínicas y de laboratorio del paciente con la presencia de delirium. Luego con las variables asociadas significativamente a delirium $(\mathrm{p}<0,05)$ se realizó el análisis de regresión logística paso a paso "stepwise". Las variables significativas luego del análisis multivariado fueron elegidas para construir el modelo de riesgo de delirium, que fue probado en su capacidad de predecir la presencia de delirium durante la hospitalización, mediante el análisis de curvas $\mathrm{ROC}^{25}$.

\section{Cohorte de validación}

Esta cohorte fue reclutada en meses sucesivos a la cohorte de desarrollo usando los mismos criterios de inclusión y exclusión. Se registró el mismo tipo de información demográfica, clínica y de laboratorio. El índice predictor de riesgo de delirium fue probado en esta cohorte y se evaluó su desempeño en curvas ROC, sensibilidad y especificidad.

\section{Resultados}

Participaron en la cohorte de desarrollo un total de 542 pacientes, con una edad promedio de $78 \pm 8$ años, $62 \%$ de sexo femenino. Se diagnóstico delirium en $35 \%$ de los pacientes. La condición de delirium se asoció a mayor edad, deterioro funcional (medido por índice de Barthel) y gravedad de la enfermedad según APACHE II $(\mathrm{p}<0,05)^{8}$. En cuanto al laboratorio, el delirium se asoció a niveles alterados de natremia $(\mathrm{Na} \leq 130$ $\mathrm{o} \geq 150 \mathrm{mmol} / \mathrm{L}$ ), hipoalbuminemia y aumento plasmático de nitrógeno ureico (Tabla 1). No se encontró una asociación significativa con velocidad de hemosedimentación, creatininemia ni con niveles plasmáticos de proteína $\mathrm{C}$ reactiva.

Tabla 1. Cohorte de desarrollo: características basales

\begin{tabular}{|c|c|c|c|c|}
\hline Variable & $\begin{array}{c}\text { Total } n \\
\text { Media (DE) } \\
n=542\end{array}$ & $\begin{array}{c}\text { Cohorte sin delirium } \\
\text { Media (DE) } \\
\mathbf{n}=\mathbf{3 5 0}\end{array}$ & $\begin{array}{c}\text { Cohorte con delirium } \\
\text { Media (DE) } \\
n=192\end{array}$ & p value \\
\hline Edad, años & $77,9 \quad(7,6)$ & $75,8 \quad(7,0)$ & $81,5 \quad(7,2)$ & $<0,001$ \\
\hline Mujeres (n,\%) & $334(61,6)$ & $215 \quad(61,4)$ & $119 \quad(62,0)$ & 0,900 \\
\hline APACHE ॥ & $10,1 \quad(4,2)$ & $9,7 \quad(4,1)$ & $10,7 \quad(4,3)$ & 0,006 \\
\hline Índice de Comorbilidad Charlson & $1,7 \quad(1,6)$ & $1,6 \quad(1,6)$ & $1,8 \quad(1,6)$ & 0,141 \\
\hline Índice de Barthel (0-100) & $86,0(20,9)$ & $92,7(15,1)$ & $73,8(24,3)$ & $<0,001$ \\
\hline Datos de Laboratorio & $\mathbf{n}$ & $\mathbf{n}$ & $\mathbf{n}$ & p value \\
\hline Sodio (mEq/L) & $137,6 \quad(5,3)$ & $138,1 \quad(4,6)$ & $136,9 \quad(6,3)$ & 0,108 \\
\hline Nitrogeno ureico (BUN) (mg/dL) & $26,9(19,7)$ & $24,1(17,4)$ & $31,8(22,4)$ & $<0,001$ \\
\hline Albumina $(\mathrm{g} / \mathrm{dL})$ & $3,7 \quad(1,8)$ & $3,9 \quad(2,2)$ & $3,5 \quad(0,6)$ & 0,003 \\
\hline VHS* (mm/hr) & $35,5(28,6)$ & $34,7(28,6)$ & $36,9(28,8)$ & 0,392 \\
\hline Proteína C reactiva** (mg/dL) & $7,2 \quad(8,9)$ & $7,4 \quad(9,1)$ & $6,8 \quad(8,2)$ & 0,48 \\
\hline BUN/Creatininemia $>18$ & $64 \%$ & $58,2 \%$ & $74,2 \%$ & $<0,001$ \\
\hline Sodio $(\leq 130 / \geq 150)$ & $8,4 \%$ & $5,7 \%$ & $13,1 \%$ & 0,003 \\
\hline
\end{tabular}

*Velocidad de hemosedimentación. ** Proteína $C$ reactiva, valor normal <0,5. 


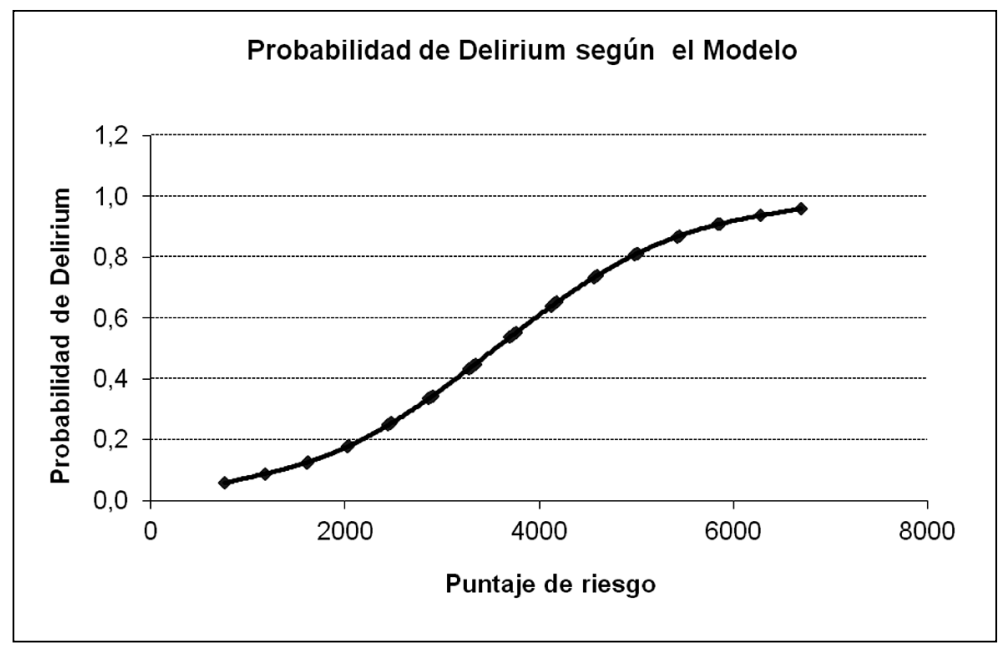

Figura 1. Probabilidad de Delirium según el modelo.
El análisis multivariado muestra que las variables independientemente asociadas a delirium sólo fueron edad avanzada y deterioro funcional, medido a través del índice de Barthel $(\mathrm{p}<0,005)$. Con estas variables se construyó el modelo capaz de predecir la presencia de delirium en algún momento de la hospitalización.

\section{Modelo de predicción de delirium: \\ (83 $x$ edad (años) - (43 $x$ Indice Barthel)}

El modelo de predicción muestra que a mayor puntaje, mayor es el riesgo de desarrollar delirium (Figura 1).

El desempeño de este modelo en la curva ROC, mostró un área bajo la curva de 0,80 (IC 0,77-0,85) en el sistema de estratificación de riesgo (Figura 2). Para predecir el desarrollo de delirium, se definieron dos puntos de cortes que diferencian grupos de bajo riesgo de delirium $(<1.700)$, riesgo intermedio (entre 1.700 y 3.000 ) y alto riesgo (sobre 3.000). El desempeño de esta clasificación se muestra en la Tabla 2. La prevalencia de delirium en cada grupo fue: $8 \%$ bajo riesgo, $23 \%$ riesgo intermedio y $69 \%$ de alto riesgo $\left(\chi^{2}, \mathrm{p}<0,001\right)$.

El modelo fue validado en una cohorte de 85 pacientes que cumplían con iguales criterios de ingreso y exclusión. La edad promedio de esta cohorte fue de 78,2 \pm 8 años y el índice de Barthel promedio fue 84,1 (DE 24,6). Estos valores no fueron estadísticamente diferentes a los de la cohorte de desarrollo (prueba $t \mathrm{p}>0,05)$. El laboratorio general (sodio, albúmina, creatinina, nitrógeno ureico y proteína $\mathrm{C}$ reactiva plasmáticos) tampoco se diferenció en forma significativa de la cohorte inicial.

En esta cohorte, 32 pacientes presentaron delirium durante su hospitalización $(37,6 \%)$, las prevalencias según el grupo de riesgo fueron: bajo riesgo $4,8 \%$, intermedio $34,3 \%$ y alto $65,5 \%$ respectivamente $\left(\chi^{2}, \mathrm{p}<0,01\right)$. El desempeño del modelo para predecir delirium en la cohorte de validación mediante la curva ROC mostró un área bajo la curva de 0,83 (IC 0,73-0,92) (Figura 2).

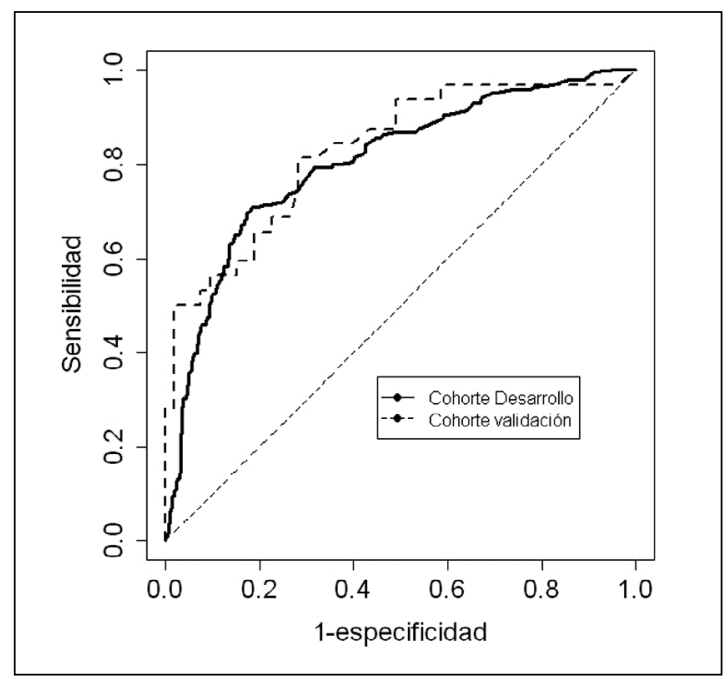

Figura 2. Desempeño del modelo de predicción en curvas ROC en cohortes de desarrollo y validación. 
Tabla 2. Desempeño del modelo de predicción de delirium en las diferentes cohortes

\begin{tabular}{|c|c|c|c|c|c|}
\hline \multirow[t]{2}{*}{ Grupo riesgo } & \multirow[t]{2}{*}{ Puntaje } & \multicolumn{2}{|c|}{ Cohorte desarrollo } & \multicolumn{2}{|c|}{ Cohorte validación } \\
\hline & & n/n (\%) & $\mathbf{R R}^{*}$ & n/n (\%) & $\mathbf{R R}^{*}$ \\
\hline Bajo & $<1.700$ & $9 / 112 \quad(8,0)$ & 1 & $1 / 21(4,8)$ & 1 \\
\hline Intermedio & $1.700-3.000$ & $55 / 243(22,6)$ & $2,8(1,4-5,5)$ & $12 / 35(34,3)$ & $7,2(1,01-51,4)$ \\
\hline Alto & $>3.000$ & $125 / 181(69,1)$ & $8,6(4,5-16,2)$ & $19 / 29(65,5)$ & $13,8(2,0-94,9)$ \\
\hline
\end{tabular}

*Riesgo relativo de delirium en el grupo de riesgo intermedio y alto, comparado al grupo de bajo riesgo usando el modelo de predicción de riesgo.

\section{Discusión}

Este estudio presenta un modelo simple y práctico que permite estratificar los pacientes de edad avanzada según su riesgo de presentar delirium durante la hospitalización, utilizando los parámetros: edad y funcionalidad previa. Con ello, logra predecir el riesgo conjunto de presentar delirium tanto incidente como prevalente.

El diseño del estudio se llevó a cabo en dos cohortes prospectivas (desarrollo y validación). En la primera se identificaron las variables independientes asociadas a delirium y con ellas se construyó el modelo. En la cohorte de validación se confirmó su capacidad predictiva y se establecieron puntos de corte para discriminar los grupos. Los valores del modelo menores de 1.700 se correlacionaron con presencia de delirium en $10 \%$ de los casos, por otro lado, aquellos valores mayores de 3.000 con $65 \%$, lo que apoya su utilidad práctica.

El modelo propuesto se basa en antecedentes que son simples de obtener. La edad ha sido identificada como uno de los principales factores de riesgo de delirium, así como también el deterioro de la funcionalidad ${ }^{26,27}$. La funcionalidad corresponde a la capacidad del individuo de realizar actividades de la vida cotidiana. Actualmente, es considerada información fundamental en el manejo y evaluación de personas mayores, dada su relevancia pronóstica y su asociación con deterioro cognitivo ${ }^{28}$. Existen distintas escalas que miden funcionalidad, siendo el índice de Barthel una de las más usadas y validadas, dada su fácil aplicación, reproducibilidad y confiabilidad ${ }^{29}$. Los datos requeridos para su aplicación pueden ser entregados por un cuidador en el momento del ingreso mediante un simple cuestionario.

Los modelos de predicción previamente publicados también se basan en factores de riesgo de delirium, que incluyen entre otros: edad, evaluación cognitiva, agudeza visual, deshidratación, gravedad de la enfermedad y polifarmacia. Sin embargo, estos modelos no han logrado traspasarse a la práctica clínica habitual ${ }^{17,19,20}$, posiblemente ha influido que estas evaluaciones requieren personal entrenado y un tiempo no menor de aplicación, siendo ambos recursos escasos en el momento del ingreso a los hospitales generales. Además, estos modelos buscan detectar sólo el delirium incidente, por lo que están diseñados para ser aplicados en pacientes sin delirium. En contraste con ellos, nuestro modelo incorpora a todos los pacientes, incluyendo aquellos que tengan deterioro cognitivo previo y aquellos que ingresen con delirium. Esto es una diferencia fundamental, considerando que hasta un tercio de los pacientes ingresan con delirium ${ }^{2}$ y que existe un alto grado de subdiagnóstico de ambas condiciones, tanto del deterioro cognitivo como del delirium ${ }^{4,30}$. Esto le permite discriminar el nivel de riesgo de todos los pacientes en el momento de admisión, para así derivarlos a unidades con implementación de estrategias de prevención y manejo precoz de los casos de delirium, como recomienda la literatura ${ }^{10}$.

Una limitación potencial de nuestro estudio es que no se evaluaron algunos factores de riesgo conocidos tales como deprivación sensorial, uso de fármacos de riesgo, abuso de sustancias ni malnutrición. A pesar de ello, a nuestro parecer, el modelo construido se basa en los dos de los principales factores de riesgo, lo que se demuestra en la potencia para predecir delirium en la cohorte de validación, y se gana en simplicidad de uso. Cabe destacar que estos hallazgos no son extrapolables a pacientes quirúrgicos ni de unidades de cuidados intensivos, requiriéndose futuros estudios en estas áreas.

Entre las fortalezas de este estudio, destacan la 
Modelo de predicción clínica de delirium - M. Carrasco et al

naturaleza prospectiva de la metodología empleada, una de las cohortes más grandes de pacientes con delirium estudiadas a la fecha y una cohorte de validación independiente a la de desarrollo. A lo anterior se agrega, dada las escasas exclusiones, que los pacientes ingresados representan pacientes "de la vida real" de los servicios de medicina hospitalarios. Una tercera fortaleza, es la naturaleza pragmática del modelo de riesgo, ya que considera variables que no dependen del delirium y no requieren de exámenes de laboratorio más exhaustivas, por lo que puede ser administrado incluso por personal administrativo en el momento del ingreso con el fin de estratificar a los pacientes según su riesgo de delirium.

Para que este sistema de estratificación de pacientes logre el beneficio esperado, se requiere que los hospitales generales desarrollen modelos de atención integral con protocolos de prevención, detección precoz y manejo adecuado de los casos de delirium. Creemos que este modelo es una herramienta útil para mejorar el cuidado de las personas mayores hospitalizadas y contribuye a un mejor manejo del delirium.

\section{Referencias}

1. Lipowski ZJ. Delirium (acute confusional states). JAMA 1987; 258 (13): 1789-92.

2. Siddiqi N, House A. Delirium: an update on diagnosis, treatment and prevention. Clinical Medicine 2006; 6 (6): 540-3.

3. Young J, Leentjens AF, George J, Olofsson B, Gustafson Y. Systematic approaches to the prevention and management of patients with delirium. J Psychosomat Res 2008; 65 (3): 267-72.

4. Carrasco M, Hoyl T, Marin PP, Hidalgo J, Lagos C, Longton $\mathrm{C}$, et al. [Delirium in Chilean elderly inpatients: an overlooked problem]. Rev Med Chile 2005; 133 (12): 1449-54.

5. McCusker J, Cole MG, Dendukuri N, Belzile E. Does delirium increase hospital stay? J Am Geriatr Soc 2003; 51 (11): 1539-46.

6. Inouye SK, Rushing JT, Foreman MD, Palmer RM, Pompei P. Does delirium contribute to poor hospital outcomes? A three-site epidemiologic study. J Gen Intern Med 1998; 13 (4): 234-42.

7. McCusker J, Cole M, Dendukuri N, Belzile E, Primeau F. Delirium in older medical inpatients and subsequent cognitive and functional status: a prospective study.
CMAJ 2001; 165 (5): 575-83.

8. González M, Martínez G, Calderon J, Villarroel L, Yuri F, Rojas C, et al. Impact of delirium on short-term mortality in elderly inpatients: a prospective cohort study. Psychosomatics 2009; 50 (3): 234-8.

9. Meagher DJ. Delirium: optimising management. BMJ 2001; 322 (7279): 144-9.

10. Leentjens AF, Rundell J, Rummans T, Shim JJ, Oldham $\mathrm{R}$, Peterson L, et al. Delirium: An evidence-based medicine (EBM) monograph for psychosomatic medicine practice, comissioned by the Academy of Psychosomatic Medicine (APM) and the European Association of Consultation Liaison Psychiatry and Psychosomatics (EACLPP). J Psychosomat Res 2012; 73 (2): 149-52.

11. Han CS, Kim YK. A double-blind trial of risperidone and haloperidol for the treatment of delirium. Psychosomatics 2004; 45 (4): 297-301.

12. Gentric A, Le Deun P, Estivin S. [Prevention of delirium in an acute geriatric care unit]. Rev Med Interne 2007; 28 (9): 589-93.

13. Inouye SK. Predisposing and precipitating factors for delirium in hospitalized older patients. Dement Geriatr Cogn Disord 1999; 10 (5): 393-400.

14. Marcantonio ER, Flacker JM, Wright RJ, Resnick NM. Reducing delirium after hip fracture: a randomized trial. J Am Geriatr Soc 2001; 49 (5): 516-22.

15. Kalisvaart CJ, Vreeswijk R, de Jonghe JF, Milisen K. [A systematic review of multifactorial interventions for primary prevention of delirium in the elderly]. Tijdschrift voor Gerontologie en Geriatrie 2005; 36 (6): 224-31.

16. Kalisvaart KJ, de Jonghe JF, Bogaards MJ, Vreeswijk R, Egberts TC, Burger BJ, et al. Haloperidol prophylaxis for elderly hip-surgery patients at risk for delirium: a randomized placebo-controlled study. J Am Geriatr Soc 2005; 53 (10): 1658-66.

17. Inouye SK, Viscoli CM, Horwitz RI, Hurst LD, Tinetti ME. A predictive model for delirium in hospitalized elderly medical patients based on admission characteristics. Ann Intern Med 1993; 119 (6): 474-81.

18. Inouye SK, Charpentier PA. Precipitating factors for delirium in hospitalized elderly persons. Predictive model and interrelationship with baseline vulnerability. JAMA 1996; 275 (11): 852-7.

19. Pompei P, Foreman M, Rudberg MA, Inouye SK, Braund V, Cassel CK. Delirium in hospitalized older persons: outcomes and predictors. J Am Geriatr Soc 1994; 42 (8): 809-15.

20. O'Keeffe ST, Lavan JN. Predicting delirium in elderly patients: development and validation of a risk-stratification model. Age Ageing 1996; 25 (4): 317-21. 
21. Knaus WA, Draper EA, Wagner DP, Zimmerman JE. APACHE II: a severity of disease classification system. Crit Care Med 1985; 13 (10): 818-29.

22. Deyo RA, Cherkin DC, Ciol MA. Adapting a clinical comorbidity index for use with ICD-9-CM administrative databases. J Clin Epidemiol 1992; 45 (6): 613-9.

23. Mahoney FI, Barthel DW. Functional Evaluation: The Barthel Index. Maryland State Medical Journal 1965; 14: 61-5.

24. González M, de Pablo J, Fuente E, Valdés M, Peri JM, Nomdedeu M, et al. Instrument for detection of delirium in general hospitals: adaptation of the confusion assessment method. Psychosomatics 2004; 45 (5): 42631.

25. Hanley JA, McNeil BJ. The meaning and use of the area under a receiver operating characteristic (ROC) curve. Radiology 1982; 143 (1): 29-36.
26. Cerejeira J, Firmino H, Vaz-Serra A, Mukaetova-Ladinska EB. The neuroinflammatory hypothesis of delirium. Acta Neuropathologica 2010; 119 (6): 737-54.

27. Elie M, Cole MG, Primeau FJ, Bellavance F. Delirium risk factors in elderly hospitalized patients. J Gen Intern Med 1998; 13 (3): 204-12.

28. Juva K, Makela M, Erkinjuntti T, Sulkava R, Ylikoski R, Valvanne J, et al. Functional assessment scales in detecting dementia. Age Ageing 1997; 26 (5): 393-400.

29. Cid-Ruzafa J, Damian-Moreno J. [Disability evaluation: Barthel's index]. Rev Esp Salud Publica 1997; 71 (2): 127-37.

30. Ely EW, Stephens RK, Jackson JC, Thomason JW, Truman B, Gordon S, et al. Current opinions regarding the importance, diagnosis, and management of delirium in the intensive care unit: a survey of 912 healthcare professionals. Crit Care Med 2004; 32 (1): 106-12. 